\title{
Bioinformatical Assistance of Selecting Anti-HIV Therapies: Where Do We Stand?
}

\author{
Thomas Lengauer \\ Max Planck Institute for Informatics, Saarbrücken, Germany
}

\section{Key Words}

HIV resistance $\cdot$ HIV tropism • Genotypic testing •

Phenotypic testing $\cdot$ Bioinformatic interpretation system

\begin{abstract}
In this opinion statement, we give a critical synopsis of the state-of-the-art of bioinformatic HIV resistance analysis and point out what we consider to be challenges and perspectives.
\end{abstract}

Copyright $\odot 2012$ S. Karger AG, Basel

\section{Genotypic Resistance Testing}

The high evolutionary dynamics of HIV afford the virus a multitude of escape paths to resistance to any applied (combination) drug therapy. With the growing arsenal of anti-HIV drugs and the enormous variant space accessible via viral evolution, the manual selection of effective anti-HIV therapies becomes increasingly challenging. The problem is especially serious with therapyexperienced patients or patients suffering from side effects for whom only limited therapy options remain. For over a decade, the manual selection of combination drug therapies has involved genotypic resistance testing, whereby the viral genotype is determined via sequencing in the laboratory and the resistance phenotype is inferred from the viral genotype. Mutation tables assembled by panels of experts [1] constituted the early basis for manual interpretation. Although their use has significantly improved the success rate of therapy switches [2], these tables have two disadvantages: first, they have limited expressiveness since they cannot represent complex interactions between mutations; second, they are based on a consensus between experts rather than on an arguably more detailed and objective quantitative analysis of clinical data. The first drawback has been removed with the introduction of rules-based systems (often somewhat misleadingly called interpretation algorithms) that apply up to hundreds of more or less complex rules governing viral genotype-phenotype relationships concerning resistance [3-5]. The rules are handcrafted and again result from expert consensus. At the beginning of the new millennium, a mathematically founded quantitative approach towards the analysis of HIV drug resistance emerged. This approach is based on collections of clinical and virological data comprising the experience of the viral response to combination anti-HIV drug therapies. Statistical prediction models can be trained on such data that afford quantitative predictions of the level of HIV resistance against individual drugs, based on the viral genotype. The effectiveness of combination drug therapies is estimated on the basis of such predictions - for all available drugs - either manually [6], or by means of simple information-joining procedures, e.g. adding the resistance scores for each drug comprising the therapy [4].

\section{KARGER}

Fax +4161306 1234

E-Mail karger@karger.ch

www.karger.com (c) 2012 S. Karger AG, Basel

$0300-5526 / 12 / 0552-0108 \$ 38.00 / 0$

Accessible online at: www.karger.com/int
Thomas Lengauer

Max Planck Institute for Informatics Campus E1 4

DE-66123 Saarbrücken (Germany)

E-Mail lengauer@mpi-inf.mpg.de 
Models of this sort have been crafted predicting the resistance of HIV to the classic antiviral drugs, nucleotide reverse transcriptase inhibitors (NRTIs), nonnucleotide reverse transcriptase inhibitors (NNRTIs) and protease inhibitors (PIs) [6], as well as predicting viral tropism, which is a relevant viral phenotype in the context of administering certain drugs that block viral cell entry [7]. Genotypic resistance testing based on computer-assisted interpretations of the viral genotype has reached the bedside today, i.e. it is routinely applied in clinical practice - be it based on expert rules or on statistical models - and is recommended by expert guidelines for anti-HIV therapy [8-13] $]^{1}$. Models based on rules determined by experts are preferred if there are insufficient clinical data for statistical modeling, e.g. for drugs that have just recently entered the marketplace. It has been shown that, if sufficient clinical data are available, statistical models outperform rules-based models in terms of predictive power [14]. Despite this, predictions based on experts' rules are generally still attractive because they are easily interpretable. However, statistical models afford interpretation of the prediction as well, e.g. the contribution of individual mutations towards strengthening or weakening resistance [6]. In the case of predicting viral tropism, no sophisticated expert rules exist because the relevant region of the viral genome, the V3 loop of the viral surface protein gp120, is short and highly variable. In this case, bioinformatic predictions are particularly in demand. As a case in point, the server geno 2 pheno ${ }_{[\text {coreceptor }}{ }^{2}$ predicting viral tropism using a statistical model receives about 10,000 queries per month.

\section{Estimation of Therapy Effectiveness}

Research on the bioinformatic assistance of anti-HIV therapy selection has gone far beyond interpreting the viral genotype as regards the viral resistance to individual drugs. Several bioinformatic systems have been developed that follow sophisticated approaches to predicting the effectiveness of drug combinations. This extends genotypic resistance testing in two directions. First, the viral phenotype no longer comprises viral response to in- dividual drugs in vitro but to drug combinations in vivo. Second, the analysis not only comprises the phenotype of the virus currently harbored by the patient but also projects the path of viral evolution to resistance - either implicitly or explicitly - and thus attempts to infer how the virus will react in the future. By assessing a whole suite of candidate therapies at a time, such a system can rank these with respect to their estimated probability of success and suggest highly promising drug combinations. The first such system that became generally available was THEO (Therapy Optimization) from the geno2pheno suite $[15,16]$, followed by the EuResist interpretation engine [17] and, more recently, by an offer from the HIV Resistance Response Database Initiative [18].

Validation studies by the groups developing these systems consistently show that such systems outperform simplistic approaches towards assessing therapy effectiveness that are based on classical genotypic resistance testing. However, an informative comparison between the systems is not available due to a lack of generally agreed notions of therapy success, the diversity of validation procedures and a lack of uniform benchmark data sets. While the predictive power of systems predicting therapy effectiveness is remarkable, such systems have not been generally accepted for use in clinical practice, i.e. their use has been limited and focal. We believe that obstacles in the way of the widespread clinical use of such systems are 4 -fold:

(1) Such systems are still in early development. A clear view on exactly what additional information to provide to them besides the viral genotype, e.g. in terms of demographic patient data, clinical parameters, therapy history or patient genotype (e.g. HLA type) is just emerging. Several approaches for providing information on patient history to the system have been investigated $[17,19]$, some of them even affording the omission of explicit information on the viral genotype $[20,21]$. Furthermore, sampling based on new-generation sequencing will afford a higher-resolution image of the viral population harbored by the patient [22]. Without a doubt, further development in these directions will result in the increased predictive power of the resulting systems.

\footnotetext{
1 UK BHIVA Guidelines for viral tropism testing: http://www.bhiva.org/Tropism.aspx

US DHHS Guidelines for anti-HIV therapy: http://www.aidsinfo.nih.gov/ContentFiles/AdultandAdolescentGL.pdf

German-Austrian DAIG-ÖAG Guidelines for viral tropism testing (in German):

http://www.daignet.de/site-content/hiv-therapie/leitlinien-1/Leitlinien\%20zur\%20Topismus_Testung\%20Stand\%20Juni\%202009.pdf German-Austrian DAIG-ÖAG: Antiretroviral Therapy of HIV Infection - German Austrian Guidelines (Sep 2008) [28].

2 http://coreceptor.bioinf.mpi-inf.mpg.de
} 
(2) Such systems infer the effectiveness of a therapy from data that are fraught with substantial representational, regional and temporal biases. Clinical databases typically comprise a few blockbuster drug combinations that are applied very frequently and then a large number of 'outlier' combinations that have only been very rarely applied. Many drug combinations, even quite feasible ones, are not represented in the database at all. Furthermore, traditions in composing drug therapies differ between different countries and regions and change over time. Statistical procedures have to take into account such biases, lest they be considered subjective in the same way that the decisions of a panel of experts can be $[23,24]$.

(3) Such systems have to be thoroughly validated in comparison to each other and also to competing approaches. The high dimensionality of the variant space both in terms of genotypic variants and in terms of therapy options requires a specially adapted innovative study design.

(4) The predictions afforded by such systems are difficult to interpret. A score resulting from a geometric analysis in high-dimensional Euclidean space or from a Bayesian probabilistic model cannot easily be made plausible to the medical user. We think that this aspect of the technology is frequently given insufficient attention. Much thought has to be given to rendering predictions comprehensible for the user. One approach could be to cast knowledge extracted from statistical analysis into rules which are then applied to the genotype. This may raise interpretability at the cost of sacrificing accuracy; however, in our experience users are quite willing to sacrifice some predictive power in exchange for more interpretable output.

\section{Rediscovery versus Discovery}

In this section we distinguish between two types of bioinformatic inference. In rediscovery we aim at identifying - albeit potentially highly complex - patterns in previously observed data, and on the basis of such patterns, making predictions on unseen data. In effect, we are rediscovering the same patterns in unseen data that we have previously identified in observed data. Two examples for bioinformatic rediscovery are template-based protein structure prediction, where we model the 3-dimensional structure of an unseen protein, the target protein, on the basis of a previously observed structure of another, generally related protein, the template protein
[25]. Thus, template and target protein essentially have the same structure. In HIV resistance analysis, we predict the resistance of a yet unseen viral genotype on the basis of mutational patterns that have been strongly correlated with the same kind of resistance on previously observed viral genotypes.

Rediscovery is to be contrasted with discovery, a process in which we make bioinformatic predictions of structures or processes that have not been observed before, not even in a similar form. In protein structure prediction, this type of inference is called de novo structure prediction. Here, we assign previously unobserved 3-dimensional structures to protein sequences. One approach to this is by simulating the process of protein folding based on physicochemical laws. This approach has not been very successful due to the complexity of the problem. To date, the most successful approach to de novo protein structure prediction reduces the problem to a rediscovery problem, by putting together the novel protein structure from structural parts that have been observed before in other proteins [26]. This is an example of the general phenomenon that bioinformatic discovery (1) remains a great challenge and (2) can become much more accessible by integrating it with and reducing it to bioinformatic rediscovery.

We intend to follow the same line of thought in the bioinformatic assistance of anti-HIV therapy selection. We purport that today most of this assistance is based on rediscovery, namely the rediscovery of previously observed associations between a viral genotype and its resistance phenotype. We have no clinical-strength methods for effectively identifying new resistance mutations before we have observed them. Our analysis is also only based on clinical experience - be it in the form of expert rules or in the form of statistical analysis of clinical resistance data. We are not basing our predictions on any kind of detailed analysis of molecular workings inside the infected cell, nor on dynamic models of viral distribution inside a patient's organs. Empowering such analysis with bioinformatics is clearly a great challenge. We believe that this challenge is best addressed by integrating focused discovery elements into the general bioinformatic rediscovery procedure that is already in place today. Let us call such a discovery element a virtual phenotype. A virtual phenotype is a bioinformatic procedure that replaces a laboratory experiment which measures some phenotypic property of the virus that is informative in the context of diagnosing or treating the disease. In this setting the experiment is the discovery element - it generates new biology.

In general, however, such an experiment is too costly, time-consuming, expensive or inaccessible to be carried 
out in clinical routine. For this reason, the experiment is virtualized, i.e., a bioinformatic prediction of its outcome based on the viral genotype is crafted via a statistical model trained on a suitable large set of experimental data.

We can give several examples of virtual phenotypes that are available today. The most widely accepted is the classical drug resistance phenotype [6]. The laboratory data for virtualizing this phenotype are generated by means of phenotypic resistance testing, a laboratory procedure that assesses the resistance of the virus by in vitro cell culture assays. With bioinformatic procedures, one then crafts statistical models that virtualize this phenotype. The virtual phenotype then replaces the laboratory procedures in future predictions. Viral tropism is another phenotype that has been virtualized in the same manner [7]. The genetic barrier to drug resistance is a virtual phenotype that assesses the virus's risk of escaping to resistance from a given drug therapy [27]. In a collaborative project with partners from the University of Heidelberg (H.-G. Kräusslich and B. Müller) and the University of Cologne (R. Kaiser), we are currently working on virtualizing another phenotype related to viral tropism, based on laboratory procedures carried out at the University of Heidelberg. Here, the effectiveness of viral cell entry is measured depending on viral, cellular and drug determinants. The idea is to craft a bioinformatic procedure that infers this dependence from only the viral genotype. We expect this novel virtual phenotype to contribute to improving the accuracy of predictions of viral tropism.

A wide variety of additional virtual phenotypes are conceivable, pertaining to detailed aspects of host-virus interactions or the viral response to the host genotype, for instance. Every accessible virtual phenotype is a potential information source for the statistical inference machinery already in place for predicting therapy effectiveness. Crafting highly informative virtual phenotypes is a generally applicable approach toward strengthening the rediscovery process that is in place today with focused elements of discovery.

ment of the effectiveness of the therapy is either manually based on the prediction of viral resistance to individual drugs or performed by simple information merging procedures. First-generation systems are in widespread clinical use in the context of the generally accepted paradigm of genotypic resistance testing and the increasingly accepted paradigm of genotypic tropism testing. Second-generation systems afford a deeper analysis of both the viral resistance phenotype and the expected escape path of the virus to drug resistance. Such systems are under intense study and in focal use, but to date, have not generally been accepted. We expect that with more investment in improving the predictive power of such systems, the interpretability of their predictions and their validation, they will, in the coming years, become increasingly valuable in clinical routine. A prerequisite for their general acceptance will also be the development of concepts for the design of studies to validate them. In our opinion, HIV therapy is the spearhead of a general development in personalized medicine that is characterized by a substantial restructuring of approaches to study design in terms of the number of patient groups and therapy options. We have pointed to ways of improving the predictive power of bioinformatic systems assisting anti-HIV therapy selection and equipping them with discovery procedures that will enable us to not only treat patients better but learn more about the molecular basis of the disease.

\section{Acknowledgements}

I am grateful to Rolf Kaiser, Alejandro Pironti, Anders Sönnerborg and Maurizio Zazzi for helpful discussions. The research has been performed in the context of the projects CHAIN (EU grant HEALTH-F3-2009-223131) and Patient- and Drug-Specific Models of HIV Cell Entry (BMBF grant 0315480A).

\section{Conclusion}

Together with new drugs and new drug mechanisms, computer-assisted selection of anti-HIV therapies has propelled the progress in AIDS therapy in the past decade. We can identify two generations of computer systems in this context. First-generation systems infer a resistance phenotype from the viral genotype. The assess-

References

Our Opinion on Bioinformatic HIV

Resistance Analysis
Johnson VA, et al: Update of the drug resistance mutations in HIV-1: December 2010. Top HIV Med 2010;18:156-163.

2 Durant J, et al: Drug-resistance genotyping in HIV-1 therapy: the VIRADAPT randomised controlled trial. Lancet 1999;353: 2195-2199.

3 Rousseau MN, et al: Patterns of resistance mutations to antiretroviral drugs in extensively treated HIV-1-infected patients with failure of highly active antiretroviral therapy. J Acquir Immune Defic Syndr 2001;26: 36-43. 
4 Van Laethem K, et al: A genotypic drug resistance interpretation algorithm that significantly predicts therapy response in HIV1-infected patients. Antivir Ther 2002;7: 123-129.

5 Rhee SY, et al: Human immunodeficiency virus reverse transcriptase and protease sequence database. Nucleic Acids Res 2003;31: 298-303.

6 6 Lengauer T, Sing T: Bioinformatics-assisted anti-HIV therapy. Nat Rev Microbiol 2006;4: 790-797.

7 Lengauer T, et al: Bioinformatics prediction of HIV coreceptor usage. Nat Biotechnol 2007;25:1407-1410.

8 Gazzard BG, et al: British HIV Association guidelines for the treatment of HIV-1-infected adults with antiretroviral therapy 2008 . HIV Med 2008;9:563-608.

$\checkmark 9$ Vandekerckhove L, et al: European guidelines on the clinical management of HIV-1 tropism testing. Lancet Infect Dis 2011;11: 394-407.

10 Clumeck N, Pozniak A, Raffi F: European AIDS Clinical Society (EACS) guidelines for the clinical management and treatment of HIV-infected adults. HIV Med 2008;9:6571.

11 Vandamme AM, et al: European Recommendations for the Clinical Use of HIV Drug Resistance Testing: 2011 update. AIDS Rev 2011;13:77-108.

$\checkmark 12$ Hirsch MS, et al: Antiretroviral drug resistance testing in adult HIV-1 infection: 2008 recommendations of an International AIDS Society-USA panel. Top HIV Med 2008; 16 : 266-285.
3 Palfreeman A, et al: Testing for HIV: concise guidance. Clin Med 2009;9:471-476.

14 Altmann A, et al: Keeping models that predict response to antiretroviral therapy upto-date: fusion of pure data-driven approaches with rules-based methods. Rev Antivir Ther 2009;1:A92.

15 Altmann A, et al: Improved prediction of response to antiretroviral combination therapy using the genetic barrier to drug resistance. Antivir Ther 2007;12:169-178.

16 Altmann A, et al: Predicting the response to combination antiretroviral therapy: retrospective validation of geno2pheno-THEO on a large clinical database. J Infect Dis 2009; 199:999-1006.

17 Rosen-Zvi M, et al: Selecting anti-HIV therapies based on a variety of genomic and clinical factors. Bioinformatics 2008;24:i399i406.

18 Larder BA, et al: Clinical evaluation of the potential utility of computational modeling as an HIV treatment selection tool by physicians with considerable HIV experience. AIDS Patient Care STDS 2011;25:29-36.

19 Saigo H, et al: Learning from past treatments and their outcome improves prediction of in vivo response to anti-HIV therapy. Stat Appl Genet Mol Biol 2011;10:article 6.

20 Prosperi MC, et al: Antiretroviral therapy optimisation without genotype resistance testing: a perspective on treatment history based models. PLoS ONE 2010;5:e13753.
21 Revell AD, et al: Modelling response to HIV therapy without a genotype: an argument for viral load monitoring in resource-limited settings. J Antimicrob Chemother 2010;65: 605-607.

22 Thielen A, Lengauer T: Geno2pheno[454] - a web server for the prediction of HIV-1 coreceptor usage from next generation sequencing data. Intervirology 2012;55:113-117.

23 Bickel S, et al: Multi-task learning for HIV therapy screening; in McCallum A, Roweis S (eds): 25th International Conference on Machine Learning (ICML 2008). Helsinki, Omnipress, 2008, pp 56-63.

24 Bogojeska J, et al: Dealing with sparse data in predicting outcomes of HIV combination therapies. Bioinformatics 2010;26:20852092.

25 Dunbrack RL Jr: Homology modeling in biology and medicine; in Lengauer T (ed): Bioinformatics - From Genomes to Therapies. Weinheim, Wiley-VCH, 2007, pp 297-350.

26 Bonneau R: De novo structure prediction: methods and applications; in Lengauer $\mathrm{T}$ (ed): Bioinformatics - From Genomes to Therapies. Weinheim, Wiley-VCH, 2007, pp 389-418.

27 Beerenwinkel N, et al: Estimating HIV evolutionary pathways and the genetic barrier to drug resistance. J Infect Dis 2005;191:19531960.

28 German-Austrian DAIG-ÖAG: Antiretroviral Therapy of HIV Infection - German Austrian Guidelines (Sep 2008) (in German). Dtsch Med Wochenschr 2009;134:S4-S15. 